\title{
A low-cost IoT-based auscultation training device
}

\author{
Heri Andrianto ${ }^{1}$, Daniel Perdana Sutanto ${ }^{2}$, Yunus Adhy Prasetyo ${ }^{3}$ \\ ${ }^{1,2}$ Electrical Engineering Department, Faculty of Engineering, Universitas Kristen Maranatha, Bandung, Indonesia \\ ${ }^{3}$ Nursing Department, Sekolah Tinggi Ilmu Kesehatan Immanuel, Bandung, Indonesia
}

\begin{tabular}{l} 
Article Info \\
\hline Article history: \\
Received Apr 4, 2020 \\
Revised Oct 20, 2020 \\
Accepted Dec 5, 2020 \\
\hline Keywords: \\
Android Smartphone \\
Auscultation \\
Internet of Things \\
Phantom doll \\
Proximity sensor \\
Training device
\end{tabular}

Training device

\begin{abstract}
Auscultation training devices are needed by teachers and students in health schools to practice auscultation techniques. In this paper, a low-cost IoTbased auscultation training device has been developed using NodeMCU, four proximity sensors, metal as a stethoscope, a switch, an android smartphone, an earphone, and a phantom doll. The message queuing telemetry transport (MQTT) protocol has been used for data communication between NodeMCU and smartphones, therefore an auscultation training hardware can be used by many students who have auscultation training application on their smartphones that subscribe to topics. The results showed that an auscultation training device was able to detect a stethoscope. Auscultation training application on a smartphone successfully plays normal and abnormal breathing sounds based on subscribed topics. With a production cost of less than $15 \mathrm{USD}$, we offer an inexpensive IoT-based auscultation training device.
\end{abstract}

This is an open access article under the CC BY-SA license.

\section{Corresponding Author:}

Heri Andrianto

Department of Electrical Engineering

Universitas Kristen Maranatha

Jl. Surya Sumantri No.65, Bandung, Jawa Barat 40164, Indonesia

Email: heri.andrianto@eng.maranatha.edu

\section{INTRODUCTION}

In the world of health, there is a technique for examining patients called auscultation. The act of listening to sounds that arise in an organ (such as the lungs or heart) with the help of diagnosis and treatment is called auscultation [1, 2]. In practice, auscultation usually uses a stethoscope as a tool [3-6]. Auscultation techniques are taught by a teacher to students using clinical/field practice learning methods. The clinical/field practice learning method is the process of student interaction with patients under the guidance and supervision conducted by clinical teachers to achieve planned competencies. However, this collaboration also requires practice in situations close to reality. Clinical simulations are a comprehensive educational methodology, not technology, which enables learning through deliberate practice of high-risk events or procedures without compromising patient safety [7]. Clinical simulation is a methodology that positions students in a real context [8], intending to practice, learn, evaluating, or gaining knowledge [9]. A student must have special expertise to diagnose lung or heart disease using a stethoscope, therefore auscultation training device is needed to obtain this expertise. The auscultation training device is used by teachers and students in health schools to practice auscultation techniques.

$\mathrm{K}$. Liu et al have designed a system used to simulate lung and heart auscultation for medical students. However, this system is complicated to be integrated because it is built using several devices such as a single Chip BF518, SD Card audio, AD1934, ADA4841, SDRAM, RS232 interface, and computer [10]. A learning application that can run on smartphone devices can be an option for students to increase knowledge and skill [11]. F. Cid et al have developed a low-cost phantom prototype for auscultation in 
simulation-based training for medical processes. The auscultation process training system uses a phantom and a stethoscope that is adapted to produce body sounds. High-quality bluetooth sound sensors and systems make it possible to simulate a collection of lung and heart diseases. The sensor on the phantom is connected to a smartphone via a wireless network and uses a cellular application to configure system settings [12]. However, research conducted by F. Cid et al still uses pressure sensors and has not used internet of things (IoT) technology, which at this time IoT technology has been widely implemented in various sectors, such as in the health sector [13-16], agriculture [17], etc. In this paper, auscultation training devices based on IoT have been developed using NodeMCU, four proximity sensors, metal as a stethoscope, a switch, an android smartphone, an earphone, and a phantom doll. We also use the MQTT protocol for data communication between NodeMCU and smartphones. MQTT is one of data communication protocols in IoT technology [1822]. MQTT is a publish-subscribe delivery protocol that is suitable for lightweight applications [23]. This protocol uses a client-server architecture where the client (device) is connected to the server (MQTT broker), which in turn forwards the message to other clients who subscribe [24, 25]. MQTT is perfect for limited environments where devices have limited processing and memory resources and low network bandwidth $[26,27]$.

\section{RESEARCH METHOD}

The research method in this study consists of designing an auscultation training system architecture, developing an auscultation training device, testing an auscultation training device, and evaluating the performance of an auscultation training device. The first stage is designing an auscultation training system architecture. Figure 1 shows the IoT-based auscultation training system architecture. IoT-based auscultation training device uses NodeMCU as a controller. NodeMCU is connected to the MQTT broker via a wi-fi connection to communicate with smartphones. We also used a phantom doll where several proximity sensors were placed inside the phantom doll. Each proximity sensor will be connected to NodeMCU. Normal and abnormal breathing sound recording files are stored on the smartphone. The switch is used to select normal or abnormal breathing sounds. If the proximity sensor detects a stethoscope drum, NodeMCU will publish topics to the MQTT broker. Auscultation training application on the smartphone will subscribe to these topics, data obtained from the topic will be used to play normal or abnormal breathing sounds, therefore that breathing sounds can be heard through the earphone.

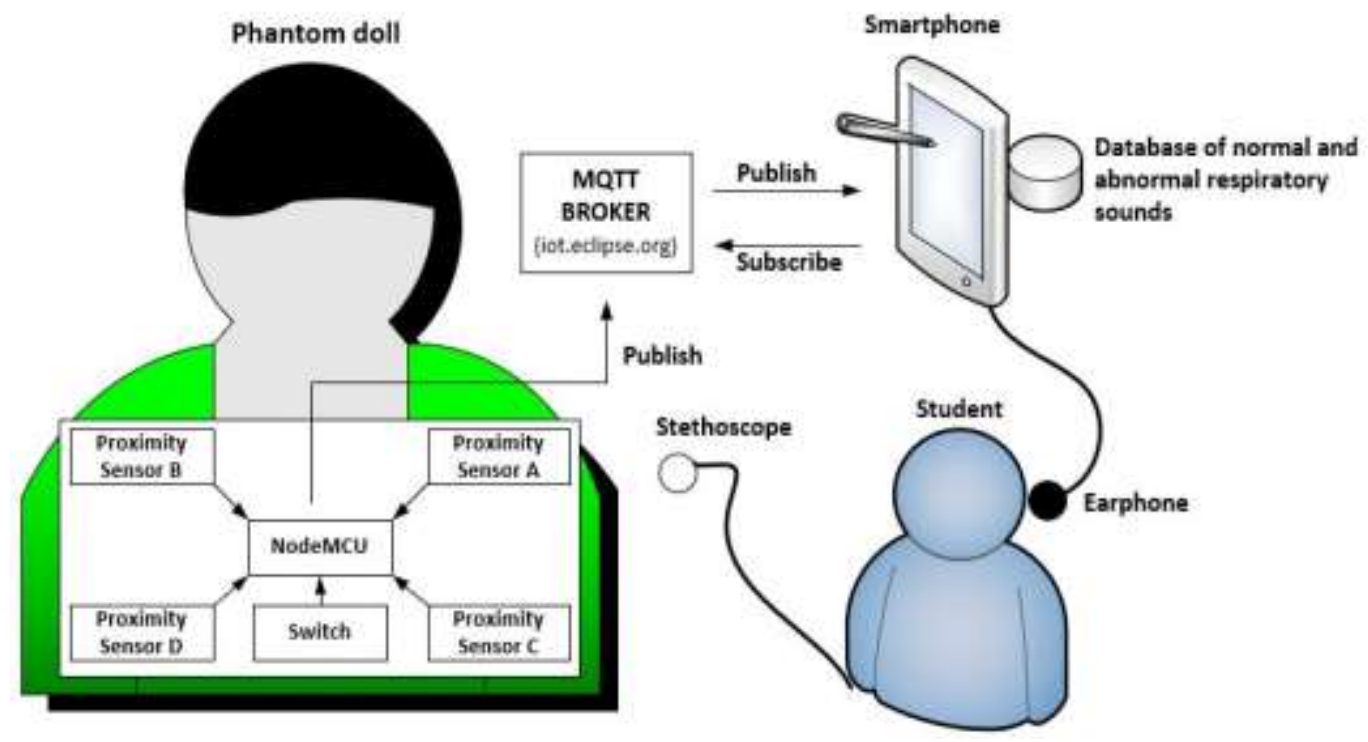

Figure 1. IoT-based auscultation training system architecture

\subsection{Hardware design}

IoT-based auscultation training device consists of NodeMCU, four proximity sensors, and a switch with the cost required for this hardware which is less than 15 USD. The proximity sensor input voltage is 12 Volt, while the NodeMCU input voltage is 3.3 Volt, therefore the proximity sensor output cannot be connected directly to the nodeMCU pin because it has different voltage levels so a signal conditioning circuit 
is needed in the form of a voltage divider circuit. Each voltage divider circuit connected to the proximity sensor is connected to the NodeMCU digital pin. The GND pin of NodeMCU and proximity sensor are connected to GND. One of the switch pins is connected to 3.3 Volt, the other pin is connected to pin D0 (GPIO16). Pin D0 (GPIO16) is also connected to one of the $10 \mathrm{k} \Omega$ resistor pins, the other pin of the $10 \mathrm{k} \Omega$ resistors is connected to GND. Pin 3V3 NodeMCU is connected to 3.3 Volt. Figure 2 shows the proximity sensor on the phantom doll. Figure 3 shows the hardware circuit design of the IoT-based auscultation training system.

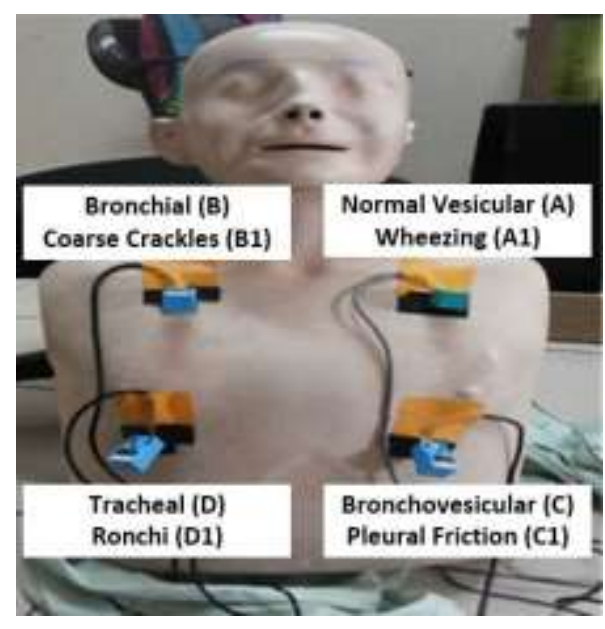

Figure 2. Proximity sensor on phantom doll

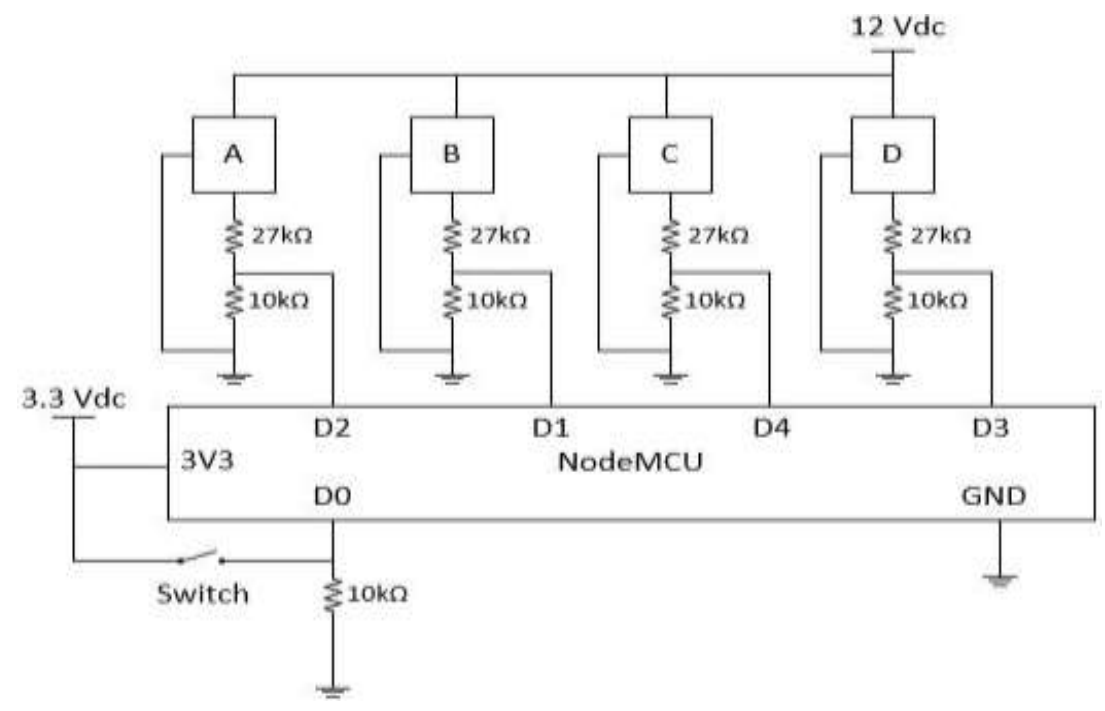

Figure 3. The hardware circuit design of IoT-based auscultation training device

\subsection{Software design}

Figure 4 shows the flowchart of the main program on NodeMCU. Proximity sensor functions to detect stethoscope drums. The function of the switch is to distinguish between normal and abnormal breathing sounds. Each proximity sensor has been configured for one normal breathing sound and one abnormal breathing sound. NodeMCU will read the pin that is connected to the proximity sensor and a switch. After that, NodeMCU will publish topics to the MQTT broker via a wi-fi connection.

Smartphone users must first choose the wi-fi connection before receiving data that has been published by NodeMCU, therefore that the data can be received well by the smartphone. The program on the smartphone checks whether there is data coming into the smartphone. If there is data coming into the 
smartphone, the data will be processed to play a sound. A database of recorded breathing sounds is stored on the smartphone. Breathing sounds will be heard in accordance with the data coming into the smartphone. For example, if the Switch is Off and the data received by the smartphone "0" with the topic "v/ breath1" then the sound of normal breathing (Normal Vesicular) will be heard on the smartphone. If the Switch is On and the data received by smartphone "0" with the topic "v / breath4" then the abnormal breathing sound (Ronchi) will be heard on the smartphone. Figure 5 shows the flowchart of the main program on smartphones.

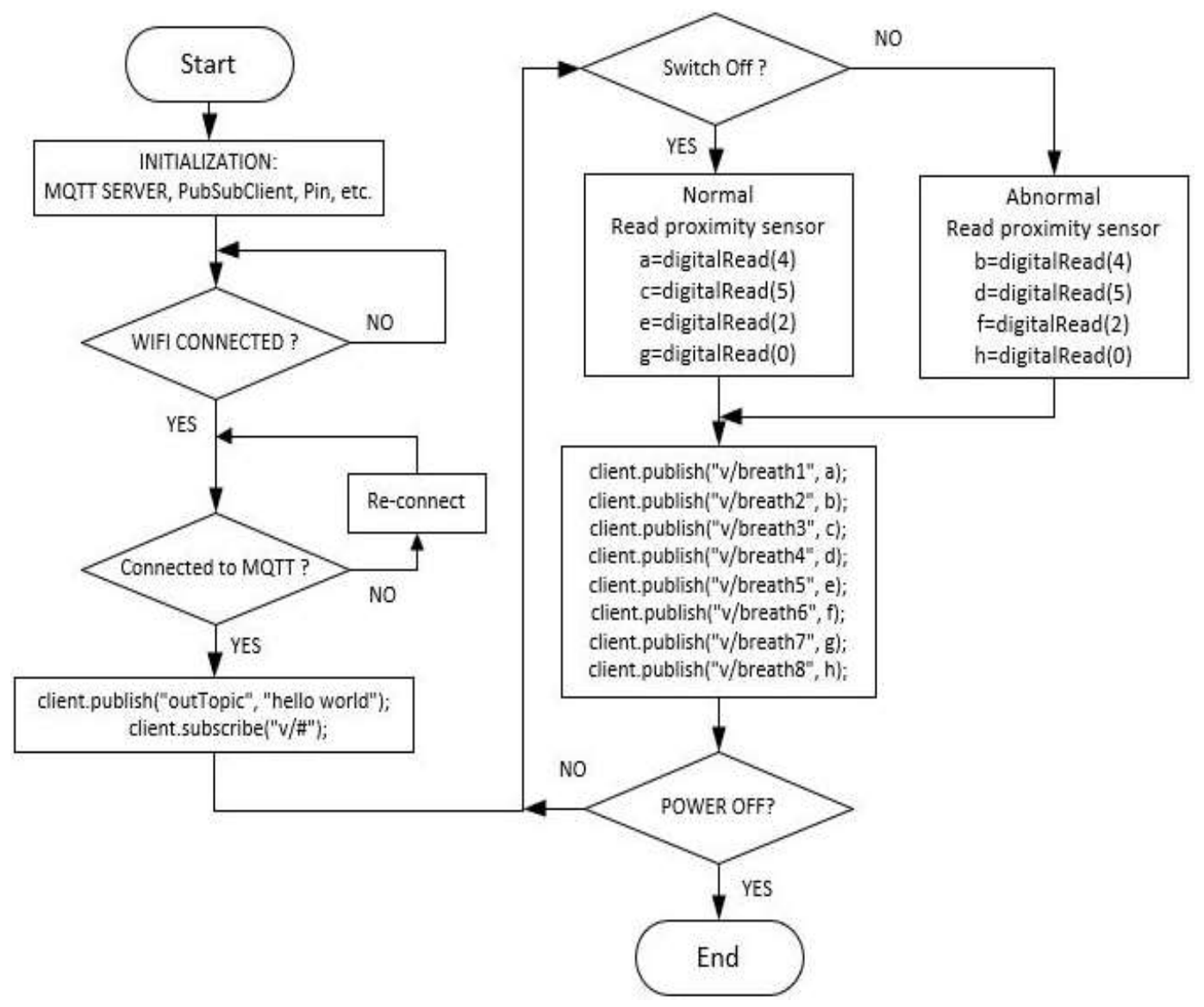

Figure 4. Main flowchart program on NodeMCU

Auscultation training application on the smartphone for normal and abnormal breathing sounds of humans is specifically used for learning auscultation techniques based on android smartphones. This smartphone application consists of three buttons (publish, connect, and disconnect) where the first button is the publish button which functions to display the words "Hello World" on the serial monitor and smartphone. The connect button functions to connect the smartphone with NodeMCU via a wi-fi connection, and the disconnect button functions to disconnect the wi-fi connection between the smartphone and NodeMCU. Two text views (Normal Breathing Sounds and Abnormal Breathing Sounds), and one textview that is specifically for displaying the writing of the selected radiobutton, for example the selected Ronchi radiobutton then the textview will display the words "Ronchi !!!!". Eight radiobutton consisting of a menu selection of normal and abnormal breathing (Tracheal Sound, Bronchial Sound, Bronchovesicular Sound, Normal Vesicular Sound, Wheezing Sound, Coarse Crackles Sound, Pleural Friction Rub Sound, and Ronchi Sound). Figure 6 shows the auscultation training application on smartphone. 


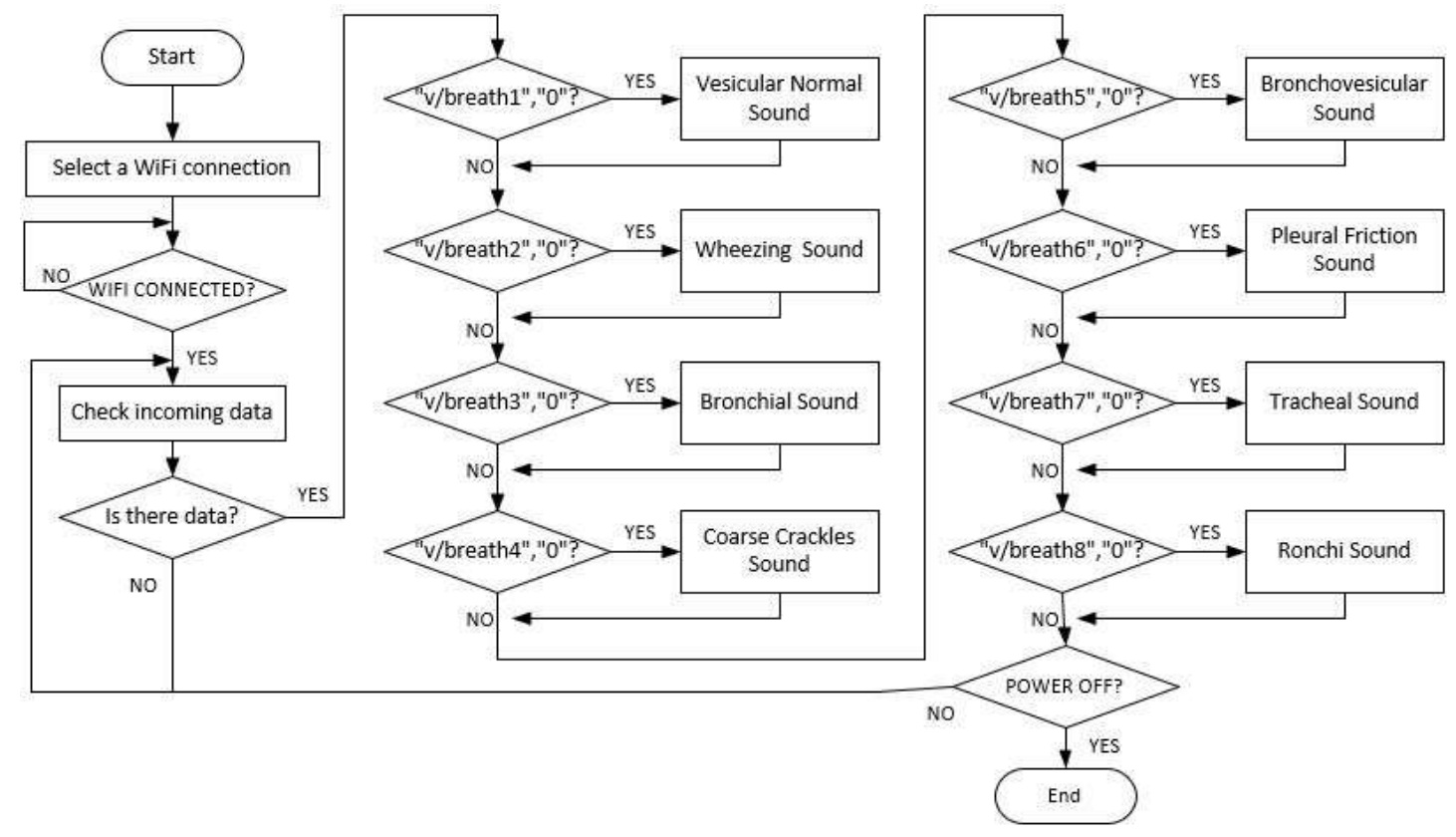

Figure 5. Flowchart of the main program on a smartphone

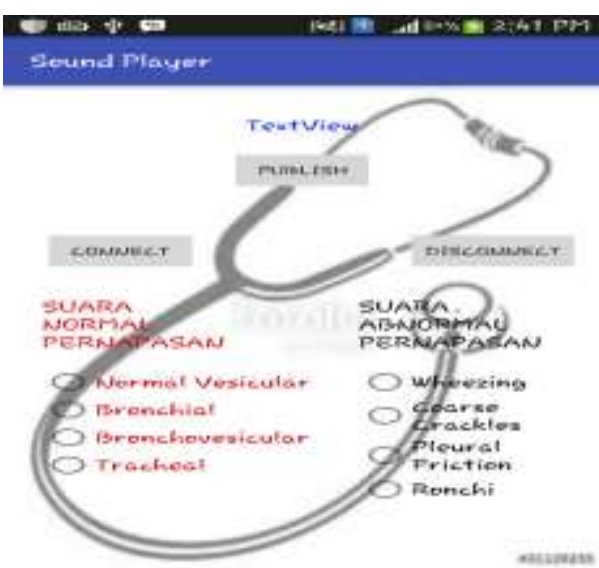

Figure 6. Auscultation training application on the smartphone

\section{RESULTS AND ANALYSIS}

Testing the connection to the wi-fi network and publish the message of stethoscope drum detection was observed on the serial monitor. Figure 7 shows that NodeMCU has successfully connected to a wi-fi network and successfully published a message. The serial monitor shows that when the proximity sensor does not detect a stethoscope drum, NodeMCU sends data "1" and if the proximity sensor detects a stethoscope drum, NodeMCU sends the data "0".
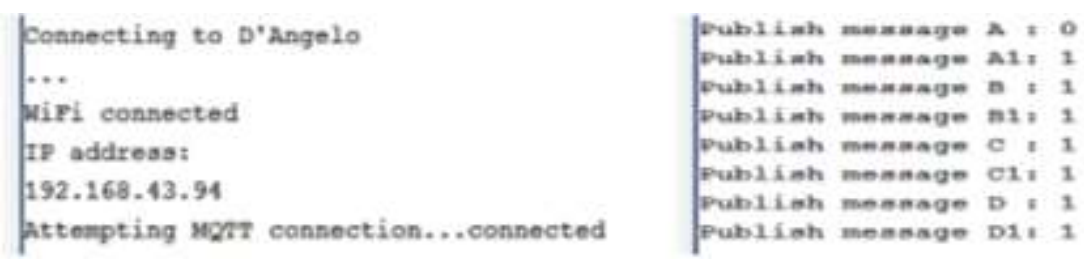

Figure 7. Testing the connection to the wifi network and stethoscope drum detection 
Communication testing between NodeMCU and smartphone has been carried out using the MyMQTT application on the smartphone. The MyMQTT application is subscribed to topics published by NodeMCU. Subscribed topics are "v / \#" which means that all topics related to "v / \#" can be seen through MyMQTT. Table 1 shows the test results, that each proximity sensor successfully detected a drum stethoscope for the OFF/ON switch position, NodeMCU successfully published topics, and the smartphone application (MyMQTT) successfully subscribed to topics published by NodeMCU.

Table 1. Testing of communication between NodeMCU and smartphone

\begin{tabular}{|c|c|c|c|c|c|c|c|c|c|c|}
\hline \multirow{2}{*}{$\begin{array}{l}\text { Switch } \\
\text { position }\end{array}$} & \multirow{2}{*}{$\begin{array}{l}\text { Proximity sensor } \\
\text { that detects a } \\
\text { stethoscope drum }\end{array}$} & \multicolumn{8}{|c|}{ Published by NodeMCU (v/breath1 to v/breath8) } & \multirow{2}{*}{$\begin{array}{c}\text { Subscribed by } \\
\text { MyMQTT }\end{array}$} \\
\hline & & $\begin{array}{c}1 \\
\text { (a) }\end{array}$ & $\begin{array}{c}2 \\
\text { (b) }\end{array}$ & $\begin{array}{c}3 \\
\text { (c) }\end{array}$ & $\begin{array}{c}4 \\
\text { (d) }\end{array}$ & $\begin{array}{c}5 \\
(\mathrm{e})\end{array}$ & $\begin{array}{c}6 \\
\text { (f) }\end{array}$ & $\begin{array}{c}7 \\
\text { (g) }\end{array}$ & $\begin{array}{c}8 \\
\text { (h) }\end{array}$ & \\
\hline OFF & A & 0 & 1 & 1 & 1 & 1 & 1 & 1 & 1 & success \\
\hline ON & A & 1 & 0 & 1 & 1 & 1 & 1 & 1 & 1 & success \\
\hline OFF & B & 1 & 1 & 0 & 1 & 1 & 1 & 1 & 1 & success \\
\hline ON & B & 1 & 1 & 1 & 0 & 1 & 1 & 1 & 1 & success \\
\hline OFF & $\mathrm{C}$ & 1 & 1 & 1 & 1 & 0 & 1 & 1 & 1 & success \\
\hline ON & $\mathrm{C}$ & 1 & 1 & 1 & 1 & 1 & 0 & 1 & 1 & success \\
\hline OFF & $\mathrm{D}$ & 1 & 1 & 1 & 1 & 1 & 1 & 0 & 1 & success \\
\hline $\mathrm{ON}$ & D & 1 & 1 & 1 & 1 & 1 & 1 & 1 & 0 & success \\
\hline
\end{tabular}

The Functional testing of auscultation training application on a smartphone is conducted when the switch is OFF, then the stethoscope is brought closer to the proximity sensor which is in position A, B, C, D, after that, it is observed which radio button is checked on the smartphone application and listen to the breathing sound that appears on the earphone. In the same way, further testing is performed when the switch is ON. Table 2 shows the test results of the auscultation training application on the smartphone. The auscultation training application on the smartphone successfully displays the checked radio button and plays a breathing sound according to the position of the switch and the stethoscope drum.

Table 2. The Functional testing of auscultation training application on smartphone

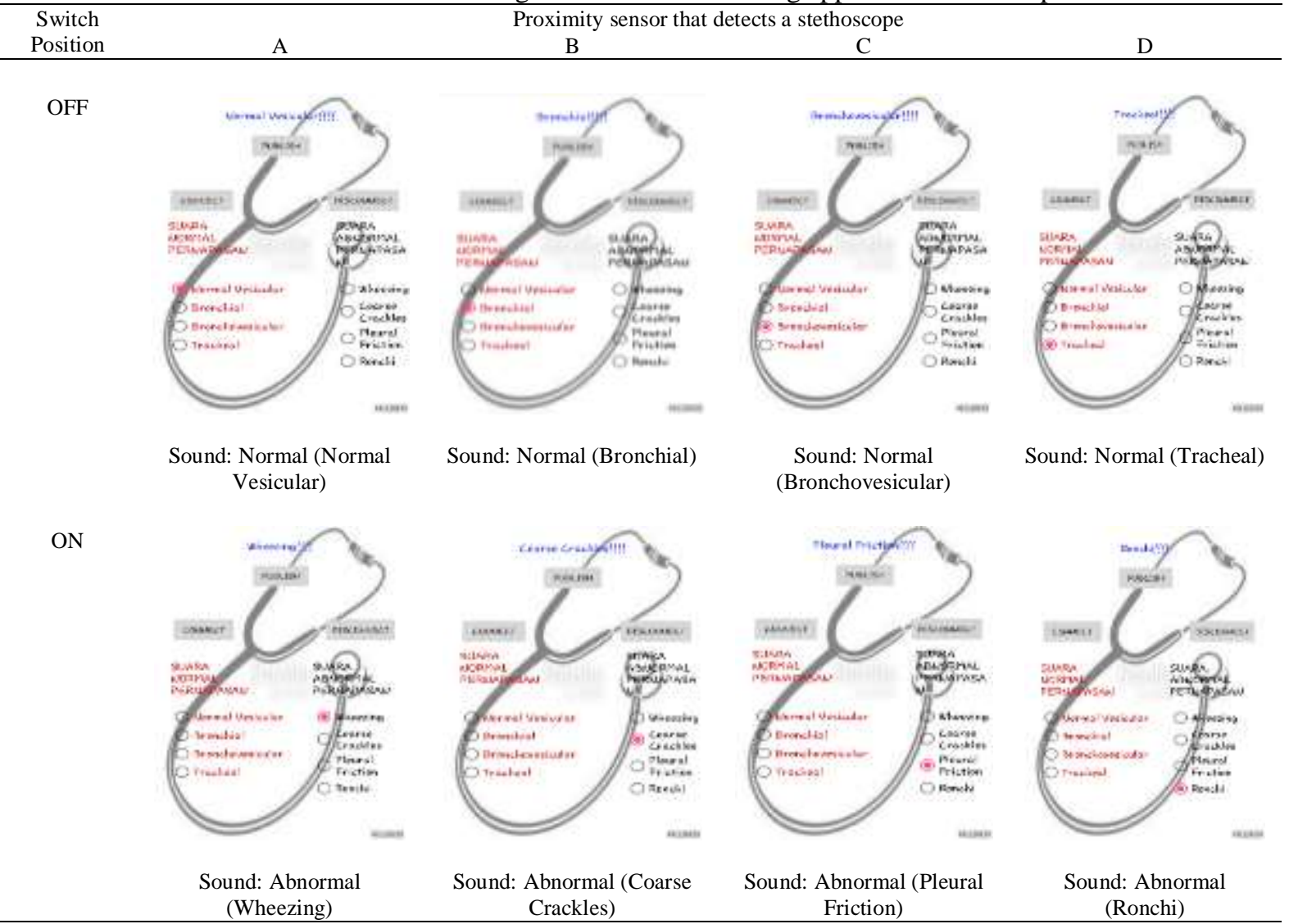




\section{CONCLUSION}

We have successfully developed a low-cost IoT-based auscultation training devices using NodeMCU, four proximity sensors, metal as a stethoscope, a switch, a phantom doll, an earphone, and an android smartphone. Each proximity sensor works well and successfully detects a stethoscope drum. NodeMCU successfully connected to the wi-fi network and successfully published topics. The Auscultation training application on smartphones successfully subscribes to topics published by NodeMCU and successfully plays normal and abnormal breathing sounds based on subscribed topics. In our future work, we will test the use of this device for the practice of auscultation techniques by students and teachers in health schools.

\section{ACKNOWLEDGEMENTS}

The authors would like to thank for the support and contributions of Universitas Kristen Maranatha and those who assisted in this research both directly and indirectly.

\section{REFERENCES}

[1] J. H. L. Serato and R. Reyes, “Automated lung auscultation identification for mobile health systems using machine learning," in Proceedings of 4th IEEE International Conference on Applied System Innovation 2018, ICASI 2018, 2018, pp. 287-290.

[2] H. Wang, J. Chen, Y. Hu, Z. Jiang, and C. Samjin, "Heart Sound Measurement and Analysis System with Digital Stethoscope," in 2009 2nd International Conference on Biomedical Engineering and Informatics, 2009, pp. 1-5.

[3] L. A. Geddes, "Birth of the stethoscope," IEEE Engineering in Medicine and Biology Magazine, vol. 24, no. 1, pp. 84-86, 2005.

[4] D. Emmanouilidou, E. D. McCollum, D. E. Park, and M. Elhilali, "Computerized Lung Sound Screening for Pediatric Auscultation in Noisy Field Environments," IEEE Transactions on Biomedical Engineering, vol. 65, no. 7, pp. 1564-1574, 2017.

[5] M. E. H. Chowdhury et al., "Real-time smart-digital stethoscope system for heart diseases monitoring," Sensors, vol. 19, no. 12, p. 2781, 2019.

[6] K. Landge, B. R. Kidambi, A. Singhal, and A. Basha, "Electronic Stethoscopes: Brief Review of Clinical Utility, Evidence, and Future Implications," Journal of the Practice of Cardiovascular Sciences, vol. 4, no. 2, pp. 65-68, 2018.

[7] A. Cheng, T. R. Lang, S. R. Starr, M. Pusic, and D. A. Cook, "Technology-enhanced simulation and pediatric education: A meta-analysis," Pediatrics, vol. 133, no. 5, pp. e 1313 - e 1323, 2014.

[8] S. George C, C. Cuadrado N, I. Solar A, J. Peralta C, H. Sanhueza G, and J. Bascuñán A, "Experiencia: aceptabilidad del uso de simulaci'on cl'inica en educaci'on m'edica: la experiencia del curso S'intesis de Conocimientos en Medicina," Revista Hospital Clinico Universitario Chile, vol. 25, no. 1, pp. 54-60, 2013.

[9] G. D. Horra, "La simulaci' on cl'inica como herramienta de evaluaci 'on de competencias en la formaci'on de enfermer'1a," Revista Reduca (Enfermer'ıa, Fisioterapia y Podolog'ı), vol. 2, no. 1, pp. 549-580, 2010.

[10] K. Liu, G. Sun, Y. Zhang, H. Ma, X. Kong, and Y. Wu, "Design of heart and lung auscultation training system based on BF518," in 2013 IEEE Global High Tech Congress on Electronics, GHTCE 2013, 2013, pp. 219-222.

[11] S. Y. Lee, P. W. Huang, J. R. Chiou, C. Tsou, Y. Y. Liao, and J. Y. Chen, "Electrocardiogram and Phonocardiogram Monitoring System for Cardiac Auscultation," IEEE transactions on biomedical circuits and systems, vol. 13, no. 6, pp. 1471-1482, 2019.

[12] F. Cid, F. Vargas, J. Melillanca, G. Schleyer, P. Gallardo, and G. Sanhueza, "An inexpensive and novel phantom prototype for auscultation in simulation-based training for medical processes," in IEEE ICA-ACCA 2018 - IEEE International Conference on Automation/23rd Congress of the Chilean Association of Automatic Control: Towards an Industry 4.0 - Proceedings, 2019, pp. 1-6.

[13] H. Ren, H. Jin, C. Chen, H. Ghayvat, and W. Chen, "A Novel Cardiac Auscultation Monitoring System Based on Wireless Sensing for Healthcare," IEEE Journal of Translational Engineering in Health and Medicine, vol. 6, pp. $1-12,2018$.

[14] N. A. A. Bakar, W. M. W. Ramli, and N. H. Hassan, "The internet of things in healthcare: An overview, challenges and model plan for security risks management process," Indonesian Journal of Electrical Engineering and Computer Science, vol. 15, no. 1, pp. 414-420, 2019.

[15] A. Muneer, S. M. Fati, and S. Fuddah, "Smart health monitoring system using IoT based smart fitness mirror," Telkomnika (Telecommunication Computing Electronics and Control), vol. 18, no. 1, pp. 317-331, 2020.

[16] P. K. Binu, K. Thomas, and N. P. Varghese, "Highly secure and efficient architectural model for iot based health care systems," in 2017 International Conference on Advances in Computing, Communications and Informatics (ICACCI), 2017, pp. 487-493.

[17] H. Andrianto, Suhardi, and A. Faizal, "Performance evaluation of low-cost iot based chlorophyll meter," Bulletin of Electrical Engineering and Informatics, vol. 9, no. 3, pp. 956-963, 2020.

[18] D. Yi, F. Binwen, K. Xiaoming, and M. Qianqian, "Design and implementation of mobile health monitoring system based on MQTT protocol," in Proceedings of 2016 IEEE Advanced Information Management, Communicates, 
Electronic and Automation Control Conference, IMCEC 2016, 2016, pp. 1679-1682.

[19] B. S. Sarierao and A. Prakasarao, "Smart Healthcare Monitoring System Using MQTT Protocol," in $20183 \mathrm{rd}$ International Conference for Convergence in Technology, I2CT 2018, 2018, pp. 1-5.

[20] S. Imane, M. Tomader, and H. Nabil, "Comparison between CoAP and MQTT in Smart Healthcare and Some Threats," in International Symposium on Advanced Electrical and Communication Technologies, ISAECT 2018 Proceedings, 2018, pp. 1-4.

[21] A. Lachtar, T. Val, and A. Kachouri, "Elderly monitoring system in a smart city environment using LoRa and MQTT," IET Wireless Sensor Systems, vol. 10, no. 2, pp. 70-77, 2020.

[22] A. Fisher, G. Srivastava, and R. Bryce, "MQTTg: An Android Implementation," in 2019 42nd International Conference on Telecommunications and Signal Processing (TSP), 2019, pp. 140-144.

[23] R. Bryce, T. Shaw, and G. Srivastava, "MQTT-G: A Publish/Subscribe Protocol with Geolocation," in $201841 s t$ International Conference on Telecommunications and Signal Processing (TSP), 2018, pp. 1-4.

[24] R. Kawaguchi and M. Bandai, "Edge Based MQTT Broker Architecture for Geographical IoT Applications," in 2020 International Conference on Information Networking (ICOIN), 2020, pp. 232-235.

[25] N. Tantitharanukul, K. Osathanunkul, K. Hantrakul, P. Pramokchon, and P. Khoenkaw, "MQTT-Topics Management System for sharing of Open Data," in 2017 International Conference on Digital Arts, Media and Technology (ICDAMT), 2017, pp. 62-65.

[26] S. Quincozes, T. Emilio, and J. Kazienko, "MQTT Protocol: Fundamentals, Tools and Future Directions," IEEE Latin America Transactions, vol. 17, no. 09, pp. 1439-1448, 2019.

[27] Q. F. Hassan, A. ur R. Khan, and S. A. Madani, Internet of Things : Challenges, Advances, and Applications. CRC Press Taylor \& Francis Group, 2017.

\section{BIOGRAPHIES OF AUTHORS}

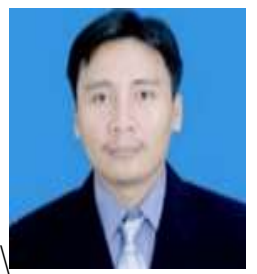

Heri Andrianto is a lecturer in electrical engineering, Maranatha Christian University, Bandung, Indonesia. He received his bachelor's degree in electrical engineering from Maranatha Christian University, Indonesia, in 2004, and his master's degree in information technology from Institut Teknologi Bandung (ITB), Bandung, Indonesia, in 2007. He is interested in the field of embedded systems, microcolntrollers, service computing, and the internet of things.

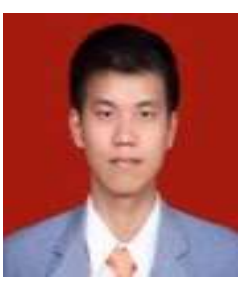

Daniel Perdana Sutanto received his bachelor's degree in electrical engineering from Maranatha Christian University, Bandung, Indonesia, in 2017.

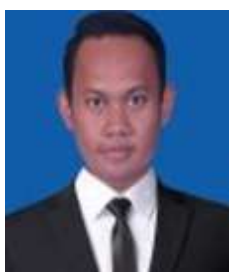

Yunus Adhy Prasetyo is a lecturer in the nursing program, Sekolah Tinggi Ilmu Kesehatan Immanuel Bandung. He finished his bachelor's degree in nursing program from STIKes Immanuel in 2007. In 2008, he completed Ners profession program from the same school. He received a government scholarship to continue his master's in nursing program from the Faculty of Nursing, Chulalongkorn University, Thailand, and completed the program in 2014. He is interested in the field of adult nursing and information technology in nursing. 